\title{
Impact of denosumab on bone mass in cancer patients
}

\author{
Ursa Brown-Glaberman \\ Alison T Stopeck \\ University of Arizona Cancer Center, \\ Tucson, AZ, USA
}

This article was published in the following Dove Press journal:

Clinical Pharmacology:Advances and Applications

3 July 2013

Number of times this article has been viewed

\begin{abstract}
Cancer therapy-induced bone loss (CTIBL) is a form of secondary osteoporosis associated with systemic chemotherapy and hormonal ablation therapy. The monitoring and treatment of CTIBL is an important component of comprehensive cancer care, especially for patients with curable disease and long life expectancies. Whereas oral bisphosphonates remain the most commonly used therapeutic option for CTIBL, additional treatment options may be required for patients who do not respond adequately or are intolerant to bisphosphonates, have renal insufficiency, or are receiving treatment with nephrotoxic medications. For these patients, denosumab, a monoclonal antibody targeting the receptor activator of nuclear factor- $\kappa \mathrm{B}$ ligand (RANKL), offers an effective and well-tolerated alternative. Several recent randomized trials have examined the use of denosumab as treatment for CTIBL associated with hormone ablation therapy for breast and prostate cancer. Recent data suggest a possible role for RANKL inhibitors in both chemoprevention and the prevention of cancer recurrence through direct effects on breast tissue and breast cancer stem cells. The outcomes of several international Phase III clinical trials currently underway will help clarify the role of denosumab in patients undergoing cancer therapy.
\end{abstract}

Keywords: denosumab, osteoporosis, osteopenia, hormone ablation therapy, cancer therapyinduced bone loss, chemotherapy

\section{Introduction}

Osteoporosis is widely recognized as a major health issue in the United States, and tremendous resources are spent screening, diagnosing, and treating this disease. According to the National Institutes of Health Consensus Development Panel on Osteoporosis, 10 million people in the United States have osteoporosis, and almost twice as many have decreased bone mass. ${ }^{1}$ Osteoporosis is defined as a decline in bone density and bone quality resulting in an increased risk for fragility fractures. ${ }^{1}$ Primary osteoporosis results from gradual bone loss with aging, whereas secondary osteoporosis is due to a variety of medical disorders, including chronic corticosteroid use, hypogonadism, alcoholism, and hyperthyroidism. Cancer therapy-induced bone loss (CTIBL) is a form of secondary osteoporosis associated with systemic chemotherapy used in the treatment of both hematologic malignancies and solid tumors and with hormone ablation therapy used in the treatment of breast and prostate cancer. The final common pathway for both primary and secondary osteoporosis is increased bone resorption in relation to new bone formation.

Diagnosis and screening for osteoporosis in men and women rely on the estimation of bone mineral density (BMD), most commonly achieved through the use of

Correspondence: Alison T Stopeck University of Arizona Cancer Center, I5I5 N Campbell Avenue, Tucson, AZ, USA 85724

Tel +I 5206262816

Email astopeck@azcc.arizona.edu 
dual-energy X-ray absorptiometry (DXA). ${ }^{1,2}$ The World Health Organization (WHO) defines osteoporosis and osteopenia on the basis of the T-score (the number of standard deviations above or below the average BMD value for young healthy white women) and the Z-score (the number of standard deviations above or below the average BMD for ageand sex-matched controls). The WHO defines osteoporosis as a T-score at least 2.5 standard deviations below normal and osteopenia as a T-score between 1.0 and 2.5 standard deviations below normal. ${ }^{3}$ These definitions were developed for estimating fracture risk in postmenopausal women and have since been extrapolated to the population at large.

The WHO-developed Fracture Risk Assessment Tool (FRAX) predicts the 10-year risk of a major fracture (defined as a fracture in the hip, clinical spine, proximal humerus, or forearm). ${ }^{4}$ The FRAX tool is customizable for use in various countries and ethnic populations and is available online at http://www.shef.ac.uk/FRAX. The FRAX score is derived from the patient's BMD or T-score at the femoral neck after adjusting for other fracture-related risk factors, including age, gender, body mass index, history of previous fracture in the patient or his/her parents, smoking status, alcohol consumption, and the presence of other secondary risks for osteoporosis. Although useful as a standardized estimate of risk, FRAX may underestimate the risk of fracture in the setting of CTIBL, particularly in breast cancer patients with bone loss associated with aromatase inhibitor (AI) therapy. ${ }^{5}$

The National Osteoporosis Foundation recommends that all men and postmenopausal women $>50$ years of age, regardless of BMD, take supplemental calcium (1200 mg daily) and vitamin D3 (800-1000 international units daily). ${ }^{6}$ This recommendation is somewhat controversial, however, as several retrospective studies have identified a potential increased risk for cardiovascular events in patients receiving calcium supplements. ${ }^{7,8}$

Lifestyle modifications, including increased physical activity and smoking and alcohol cessation, are also encouraged in the National Osteoporosis Foundation guidelines. Additional pharmacotherapeutic intervention is considered for patients with osteoporosis or osteopenia at the femoral neck or spine and a 10-year hip fracture probability $\geq 3 \%$ or a 10 -year major osteoporosis-related fracture probability $\geq 20 \%$ based on the US-adapted FRAX evaluation. ${ }^{6}$ Pharmacologic treatment options for osteoporosis in the general population include bisphosphonates, hormone replacement therapy, selective estrogen-receptor modulators (SERMs), strontium ranelate (not currently available in the United States), teriparatide, and denosumab. However, only bisphosphonates and denosumab are generally used in the treatment of CTIBL. Other pharmacologic treatment options for primary osteoporosis, including teriparatide and exogenous estrogens, are infrequently used in cancer patients because of perceived risks or associated toxicities. For example, teriparatide has been associated with an increased risk for osteosarcoma, particularly in patients who have received prior radiation therapy. Estrogen therapy is contraindicated in patients with hormone receptor-positive breast cancer and is associated with gynecomastia and thrombotic complications in men.

This manuscript focuses on the treatment of CTIBL, particularly that associated with hormone ablation therapy for the treatment of breast and prostate cancer, with denosumab, a monoclonal antibody targeting the receptor activator of nuclear factor- $\mathrm{KB}$ (RANK) ligand (RANKL).

\section{Role of estrogen and testosterone in bone turnover}

Normal bone metabolism is characterized by continual osteoclast-mediated bone resorption balanced by osteoblastmediated new-bone formation, a process predominantly regulated by the RANK-RANKL system. ${ }^{9}$ RANKL is produced by osteoblasts, bone marrow stromal cells, and other cells under the control of various proresorptive growth factors, hormones, and cytokines. Osteoblasts and stromal cells also produce osteoprotegerin (OPG), a decoy receptor, which binds to and thereby prevents RANKL from activating its receptor, RANK. In the absence of OPG, RANKL activates RANK expressed on osteoclasts and preosteoclast precursors, promoting preosteoclast recruitment, fusion into multinucleated osteoclasts, osteoclast activation, and osteoclast survival. Activated osteoclasts secrete acids and other factors that break down the bone matrix, releasing calcium and phosphate into circulation. Thus, the bone serves as the body's reservoir for calcium homeostasis.

Both estrogen receptors and androgen receptors are present in chondrocytes, bone marrow stromal cells, osteoblasts, osteoclasts, and osteoclast progenitors. ${ }^{10}$ Estrogen deficiency is a major cause of postmenopausal bone loss in women and of age-related bone loss in both sexes. Estrogen deficiency results primarily in the loss of cortical bone, which comprises more than $80 \%$ of the skeleton, and is the major contributor to overall fracture risk. ${ }^{11}$ Estrogen, acting via the estrogen receptor, stimulates osteoclast apoptosis and, conversely, suppresses osteoblast and osteocyte apoptosis. ${ }^{12}$ Cell-culture studies demonstrate 
that estrogen can also increase OPG production and in some cases suppress RANKL expression. ${ }^{9}$ Estrogen deficiency is also associated with an increase in the levels of circulating inflammatory cytokines, including tumor necrosis factor- $\alpha$ and interleukin- $1 \alpha$, which are potent stimulators of RANKL expression. ${ }^{12}$ Thus, the cumulative effect of a low-estrogen state is to increase the RANKL:OPG ratio, which results in increased osteoclast activation and survival and unbalanced bone resorption.

The role of androgens in bone homeostasis is less well defined. Testosterone is peripherally converted via the aromatase enzyme into estrogens essential for bone health in both men and postmenopausal women. ${ }^{12,13}$ Thus, decreased BMD in men undergoing androgen-deprivation therapy (ADT) is largely secondary to the resulting estrogen deficiency. Testosterone may also have direct antiresorptive effects and is important for the maintenance of bone formation. ${ }^{13}$ By increasing cortical bone thickness and preserving muscle strength, testosterone may also decrease the risk of falls and the likelihood of posttrauma fracture. ${ }^{13,14}$

\section{Bone loss with hormone ablation in cancer treatment Breast cancer}

AIs are currently the most commonly prescribed adjuvant endocrine therapy for postmenopausal women with estrogen receptor-positive breast cancer. AIs inhibit aromatase, the cytochrome P450 CYP-19 enzyme responsible for the peripheral conversion of androgens to estrogens. Commercially available options include the nonsteroidal AIs letrozole and anastrozole and the steroidal AI exemestane. Other options for adjuvant endocrine therapy in both premenopausal and postmenopausal women with breast cancer are SERMs, including tamoxifen, and in premenopausal women are oophorectomy and lutenizing hormone-releasing hormone (LHRH) agonists, including goserelin and leuprolide. AIs, LHRH agonists, and oophorectomy are associated with bone density loss, whereas SERMs increase BMD in postmenopausal women due to their proestrogenic effects on bone. Several clinical trials evaluating the use of AIs in the adjuvant setting have demonstrated a decrease in BMD with an associated increase in fractures. As an example, a subset analysis was performed in the 5-year Arimidex, Tamoxifen, Alone or in Combination (ATAC) trial in 108 evaluable postmenopausal women with breast cancer randomly assigned to anastrozole versus tamoxifen. ${ }^{15}$ Lumbar spine and total hip BMD were assessed at baseline and after 1 year, 2 years, and 5 years. Women assigned to anastrozole had a greater decrease in median BMD from baseline to year 5 at the lumbar spine ( $6 \%$ loss) and total hip (7.2\% loss), compared with women assigned to tamoxifen treatment. In this trial, women treated with tamoxifen demonstrated an increase in BMD at the lumbar spine $(2.8 \%$ gain) and total hip $(0.74 \%$ gain $) .{ }^{15}$ When the entire trial population of 6241 women was considered, significantly more fractures were observed in patients randomized to anastrozole than in patients randomized to tamoxifen (451 fractures with anastrozole versus 351 with tamoxifen; odds ratio $[\mathrm{OR}]=1.33$; $95 \%$ confidence interval $[\mathrm{CI}]=1.15-1.55$; $P<0.0001)$. However, after treatment completion, the incidence of fractures equalized between the two groups (110 anastrozole versus 112 tamoxifen; OR $=0.98 ; 95 \%$ $\mathrm{CI}=0.74-1.30 ; P=0.9) .{ }^{16}$

Many other trials have confirmed these results. A meta-analysis of seven randomized trials comprising approximately 30,000 postmenopausal women with early-stage breast cancer found that the use of AIs significantly increased the risk of fractures (OR $=1.47 ; 95 \%$ $\mathrm{CI}=1.34-1.61)$ compared with tamoxifen. ${ }^{17}$ Guidelines from the American Society of Clinical Oncology, the National Comprehensive Cancer Network (NCCN), and the European Society for Medical Oncology recommend BMD testing by DXA for all postmenopausal women taking AIs. ${ }^{18-20}$

\section{Prostate cancer}

The prolonged use of LHRH agonists in both men and women is associated with a decline in BMD. Loss of BMD can be detected in the first year of ADT in men with prostate cancer, with longer durations of therapy conferring higher risk. $^{21,22}$ In a retrospective analysis of more than 50,000 men with prostate cancer in the Surveillance, Epidemiology, and End Results database, $19.4 \%$ of men treated with ADT and surviving at least 5 years after diagnosis had a documented fracture, compared with only $12.6 \%$ of those not receiving ADT $(P<0.001) .{ }^{23}$ A statistically significant association was found between the number of doses of gonadotropinreleasing hormone received during the 12 months after diagnosis and the subsequent risk of fracture. ${ }^{23}$ NCCN guidelines currently recommend obtaining a baseline DXA scan prior to initiating ADT in men at increased risk of fracture on the basis of their FRAX score. ${ }^{24} \mathrm{~A}$ follow-up DXA scan after 1 year of therapy is suggested in higher-risk patients; however, there is currently no consensus on the optimal monitoring schedule. 


\section{CTIBL in other settings}

While men and women undergoing hormone ablation therapy are the most studied group of patients with CTIBL, other cancer patients are also at risk. For example, a populationbased cohort study of older adult patients with non-Hodgkin lymphoma treated with chemotherapy demonstrated a significantly higher risk of osteoporosis and fractures. ${ }^{25}$ The observed decrease in BMD in patients undergoing treatment with cytotoxic chemotherapy is multifactorial. Hypogonadism resulting from exposure to chemotherapeutic agents is the dominant mechanism in younger patients. ${ }^{26} \mathrm{In}$ a small study, 35 breast cancer patients with ovarian failure after adjuvant chemotherapy had significant decreases in BMD, most pronounced in the lumbar spine, at 6 months and 12 months, compared with women with preserved ovarian function. ${ }^{27}$

Cytotoxic chemotherapy agents, including methotrexate, cyclophosphamide, and doxorubicin, also have been shown to have direct inhibitory effects on bone formation in animal models. ${ }^{26,28,29}$ Ifosfamide and platinum compounds are thought to impact BMD at least in part by inducing hypophosphatemia and hypomagnesemia, respectively. ${ }^{26,30,31}$ Much of this data come from studies in children treated for acute lymphoblastic leukemia; however, there are multiple other factors contributing to osteoporosis in these patients, including failure to reach a normal peak bone mass in early adulthood, prolonged chronic illness, prolonged corticosteroid use, cranial irradiation with damage to pituitary function, and vitamin D deficiency. ${ }^{32}$

Glucocorticoids are used commonly in the treatment of hematologic malignancies and in the prevention of nausea and hypersensitivity reactions associated with chemotherapy for solid tumors. Glucocorticoids contribute to bone loss via several mechanisms, including increasing the RANKL:OPG ratio, impacting calcium balance, directly decreasing bone formation via glucocorticoid-induced apoptosis of both osteoblasts and osteocytes, and prolonging the life span of osteoclasts. ${ }^{26,33,34}$

Patients undergoing hematopoietic stem cell transplantation (SCT) are uniquely prone to osteopenia or osteoporosis. Most bone loss in SCT patients occurs during the first 3-6 months after transplant and is most severe at the femoral neck..$^{35}$ These patients are typically treated with highdose cytotoxic chemotherapy, resulting in hypogonadism. They also receive prolonged systemic corticosteroids and other immunosuppressive agents, have lengthy periods of inactivity, and are frequently vitamin-D deficient; all of which contribute to excessive bone resorption. ${ }^{26}$ SCT may also directly damage the marrow, resulting in an inability to regenerate normal numbers of osteoblastic precursors in the stromal stem cell compartment. ${ }^{36}$

\section{Treatment of CTIBL Bisphosphonates}

In addition to adequate calcium and vitamin D intake, bisphosphonates have been the mainstay of treatment for CTIBL. Although frequently prescribed, none of the bisphosphonates are specifically approved by the US Food and Drug Administration (FDA) for the treatment of CTIBL. However, data from several randomized trials have proven their effectiveness in the treatment of CTIBL in patients with breast and prostate cancer, and several bisphosphonates are Medicare compendia-approved for this indication..$^{37}$ Bisphosphonates are rapidly incorporated into bone and are released during osteoclast-mediated bone resorption, during which they impair the ability of the osteoclasts to adhere to the bony surface and inhibit further bone resorption. Bisphosphonates also decrease osteoclast progenitor development and recruitment and induce osteoclast apoptosis. Oral (PO) bisphosphonates available for the treatment of osteoporosis in the United States include alendronate, risedronate, and ibandronate, all of which have been shown in clinical trials to be effective at preventing bone loss, improving BMD, and reducing fracture risk. Intravenous (IV) bisphosphonates, including zoledronic acid, pamidronate, and ibandronate (not available in the United States), have also been shown to be effective for this indication.

The most common side effects associated with PO bisphosphonates are gastrointestinal and include reflux, esophagitis, and esophageal ulcers. Common side effects reported with IV bisphosphonates include flu-like symptoms, bone pains, and hypocalcemia. Bisphosphonates are also renally cleared and thus can cause nephrotoxicity. Consequently, they are contraindicated in patients with severe or moderate renal insufficiency (creatinine [Cr] clearance $<30-35 \mathrm{~mL} /$ minute) and should be used with caution in patients receiving other nephrotoxic medications. Rare, but serious, side effects associated with both PO and IV bisphosphonates include osteonecrosis of the jaw (ONJ) and atypical femur fractures. The full side-effect profile associated with bisphosphonate therapy was recently reviewed by McClung et al. ${ }^{38}$

Several trials have established a role for both PO and IV bisphosphonates in the treatment of bone loss associated with AI therapy in breast cancer. ${ }^{39-43}$ Similarly, bisphosphonates 
have been shown to increase $\mathrm{BMD}$ in men receiving $\mathrm{ADT}$ for prostate cancer. ${ }^{44-47}$ Of note, none of the breast and prostate cancer trials evaluating the use of bisphosphonates for the treatment and prevention of CTIBL have been powered to evaluate fracture rates as a primary outcome.

\section{Denosumab}

The pivotal role of the RANK-RANKL pathway in bone physiology renders it a prime target for the treatment of bone disease. The first commercially available drug to specifically target the RANK-RANKL pathway is denosumab, a fully human monoclonal antibody that binds and neutralizes RANKL, thereby inhibiting osteoclast function. The initial Phase I trials demonstrated that osteoclastic activity was almost completely abolished by denosumab therapy. ${ }^{48}$ However, the effect is reversible, as indicated by a rise in markers of bone turnover when the drug is cleared by the reticuloendothelial system. ${ }^{48}$ Denosumab has FDA approval under two brand names. Under the brand name Prolia ${ }^{\circledR}$ (Amgen Inc, Thousand Oaks, CA, USA), denosumab is indicated for the treatment of postmenopausal osteoporosis and CTIBL, at a dose of $60 \mathrm{mg}$ subcutaneously (SC) every 6 months. Under the brand name $\mathrm{XGEVA}^{\circledR}$ (Amgen Inc), denosumab is FDA approved for the prevention of skeletalrelated events (SREs) in patients with bone metastases from solid tumors, at a dose of $120 \mathrm{mg}$ SC every 4 weeks.

Initial trials established the efficacy of denosumab in the treatment of postmenopausal women with primary osteoporosis. In the Phase III Fracture Reduction Evaluation of Denosumab in Osteoporosis Every 6 Months (FREEDOM) trial, 7868 postmenopausal women (60-90 years of age) with osteoporosis were randomly assigned to denosumab (60 mg SC every 6 months) or placebo. ${ }^{49}$ At 3 years, the incidence of new vertebral fractures (the primary endpoint) was $2.3 \%$ in the denosumab arm versus $7.2 \%$ in the control arm, a relative-risk reduction of $68 \%(P<0.001)$. The risk of nonvertebral and hip fractures was also significantly reduced in the treatment arm.

Denosumab was also evaluated in the setting of primary osteoporosis in a Phase III, double-blind, active-controlled trial comparing the efficacy and safety of denosumab (60 mg SC every 6 months) with alendronate ( $70 \mathrm{mg}$ PO weekly) in 1189 postmenopausal women with a BMD T-score $<-2.0 .^{50}$ At 12 months, the denosumab-treated patients had a statistically significant increase in BMD at the total hip (the primary endpoint) compared with the alendronate-treated patients $(3.5 \%$ versus $2.6 \%$, respectively; $P<0.0001) .{ }^{50}$ Although this study was not powered to compare fracture rates, they were similar between the groups, with 18 fractures $(3.0 \%)$ in the denosumab group compared with $13(2.2 \%)$ in the alendronate group $(P=0.37) .{ }^{50}$

Table 1 summarizes the outcomes and fracture rates from the Phase III clinical trials evaluating denosumab in the treatment of osteoporosis, osteopenia, and CTIBL.

\section{Denosumab and CTIBL in breast cancer}

Denosumab has also been evaluated specifically in the treatment and prevention of CTIBL associated with adjuvant AI therapy in breast cancer. In a randomized, double-blind, placebo-controlled trial, 252 women with hormone receptor-positive early-stage breast cancer treated with adjuvant $\mathrm{AI}$ therapy were randomly assigned to receive placebo or denosumab (60 mg SC every 6 months). ${ }^{51}$ At enrollment, all patients were required to have evidence of low bone mass, excluding osteoporosis (lumbar spine, total hip, or femoral neck BMD corresponding to a T-score of -1.0 to -2.5$)$. Patients were also required to have 25 -hydroxyvitamin D levels $\geq 12 \mathrm{ng} / \mathrm{mL}$. Key exclusion criteria included current use of bisphosphonates and the use of antineoplastic therapy aside from AI. The primary endpoint was percentage change in the lumbar spine BMD at months 12 and 24, compared with baseline. At 12 and 24 months, lumbar spine BMD increased by $5.5 \%$ and $7.6 \%$, respectively, in the denosumab group compared with the placebo group $(P=0.0001)$. This increase was observed irrespective of the duration of prior AI or tamoxifen therapy. As in previous trials, markers of bone metabolism were rapidly suppressed. At only 252 patients, the study was not powered to see differences in fracture rates; however, the incidence of nonvertebral fractures was $6 \%$ in both the denosumab and placebo arms (Table 1). A randomized Phase III clinical trial is currently underway to determine whether, compared with placebo, denosumab will reduce the rate of first clinical fracture in women with nonmetastatic breast cancer receiving AI therapy. ${ }^{52}$

The overall incidence of grade 3 or higher adverse events (AEs) was similar between treatment groups and included AEs commonly associated with AI therapy, including arthralgia, pain in the extremities, back pain, and fatigue (Table 2). ${ }^{51}$ Significant hypocalcemia was not reported. Two deaths (one in each group) were reported, both attributed to breast cancer progression. In a subgroup analysis, an increase in BMD from baseline was seen with denosumab compared with placebo across all subgroups and skeletal sites. This increase was statistically significant, with two exceptions: (1) radial BMD in patients who received prior 


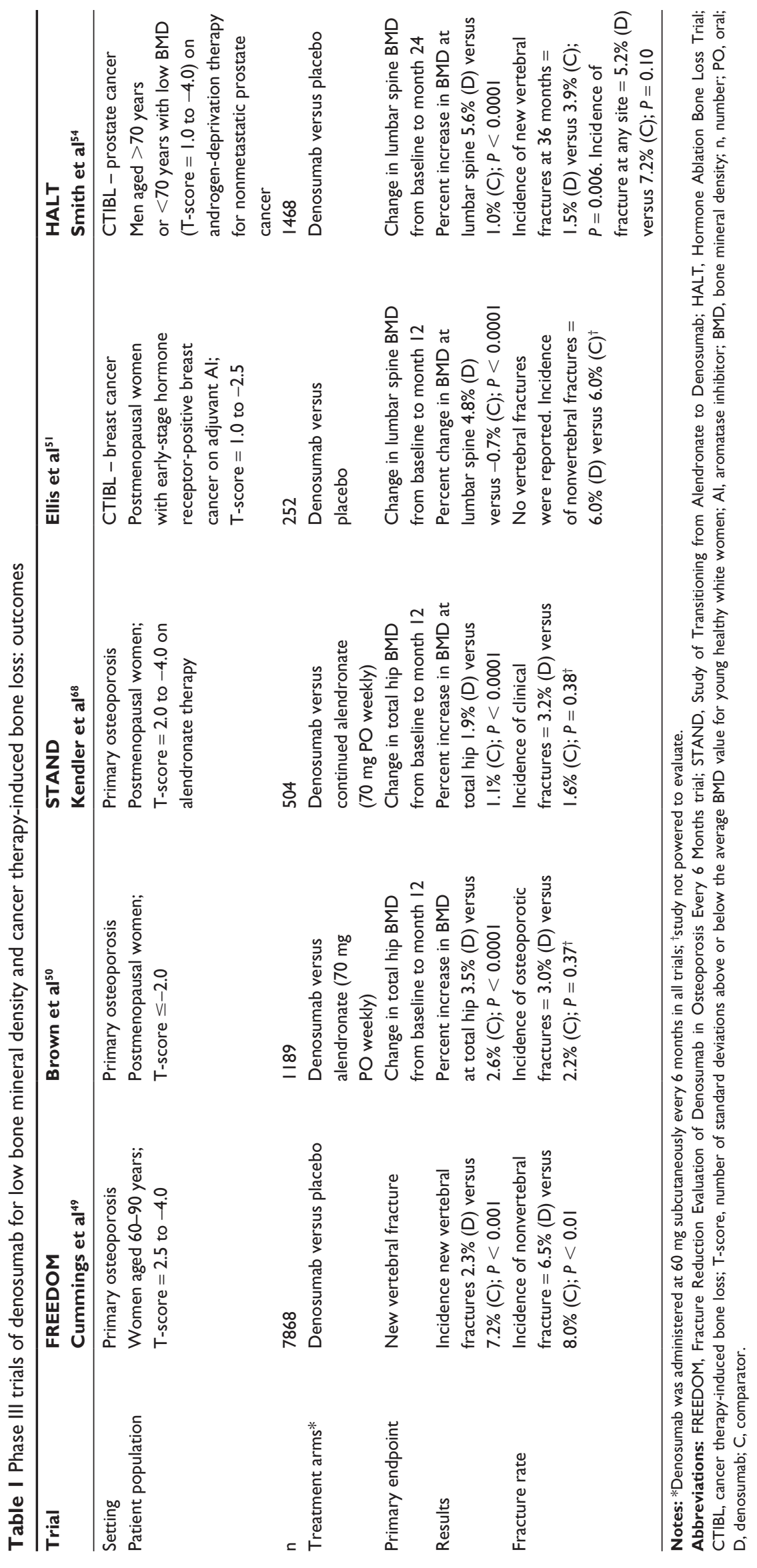


Table 2 Adverse events from denosumab trials

\begin{tabular}{|c|c|c|c|c|c|}
\hline Trial & $\begin{array}{l}\text { FREEDOM } \\
\text { Cummings et } \mathrm{al}^{49}\end{array}$ & Brown et $\mathbf{a l}^{50}$ & $\begin{array}{l}\text { STAND } \\
\text { Kendler et } \mathrm{al}^{68}\end{array}$ & Ellis et $\mathbf{a l}^{51}$ & $\begin{array}{l}\text { HALT } \\
\text { Smith et } \text { al }^{54}\end{array}$ \\
\hline Treatment arms & $\begin{array}{l}\text { Denosumab } \\
\text { versus placebo }\end{array}$ & $\begin{array}{l}\text { Denosumab versus } \\
\text { alendronate ( } 70 \mathrm{mg} \\
\text { PO weekly) }\end{array}$ & $\begin{array}{l}\text { Denosumab versus } \\
\text { continued alendronate } \\
\text { (70 mg PO weekly) }\end{array}$ & $\begin{array}{l}\text { Denosumab } \\
\text { versus placebo }\end{array}$ & $\begin{array}{l}\text { Denosumab } \\
\text { versus placebo }\end{array}$ \\
\hline \multicolumn{6}{|c|}{ AEs, denosumab versus comparator, $n(\%)$} \\
\hline Overall AEs & $\begin{array}{l}3605(92.8) \text { versus } \\
3607(93.1)\end{array}$ & $\begin{array}{l}480(80.9) \text { versus } \\
482(82.3)\end{array}$ & $\begin{array}{l}197(77.9) \text { versus } \\
196(78.7)\end{array}$ & $\begin{array}{l}\text { II } 17(90.7) \text { versus } \\
108(90.0)\end{array}$ & $\begin{array}{l}638(87.3) \text { versus } \\
627(86.5)\end{array}$ \\
\hline Serious AEs & $\begin{array}{l}\text { I004 (25.8) versus } \\
972(25 . I)\end{array}$ & $\begin{array}{l}34(5.7) \text { versus } \\
37(6.3)\end{array}$ & $\begin{array}{l}\text { I5 (5.9) versus } \\
16(6.4)\end{array}$ & $\begin{array}{l}19(14.7) \text { versus } \\
\text { II }(9.2)\end{array}$ & $\begin{array}{l}253(34.6) \text { versus } \\
222(30.6)\end{array}$ \\
\hline Hypocalcemia & 0 versus $3(0.1)$ & $I(0.1)$ versus 0 & $I(0.1)$ versus 0 & $\begin{array}{l}2(2.0) \text { versus } \\
2(2.0)\end{array}$ & $I(0.1)$ versus 0 \\
\hline Osteonecrosis of the jaw & 0 versus 0 & Not reported & Not reported & Not reported & 0 versus 0 \\
\hline Infection & $\begin{array}{l}159(4.1) \text { versus } \\
133(3.4)\end{array}$ & $\begin{array}{l}221(37.3) \text { versus } \\
207(35.3)\end{array}$ & $\begin{array}{l}\text { I I I (43.9) versus } \\
93(37.3)\end{array}$ & $\begin{array}{l}45(36.0) \text { versus } \\
40(32.0)\end{array}$ & $\begin{array}{l}43(5.9) \text { versus } \\
33(4.6)\end{array}$ \\
\hline $\begin{array}{l}\text { Cellulitis (including } \\
\text { erysipelas) }\end{array}$ & $\begin{array}{l}\mathrm{I} 2(0.3) \text { versus } \\
\mathrm{I}(<0 . \mathrm{I})^{*}\end{array}$ & Not reported & Not reported & Not reported & $\begin{array}{l}2(0.3) \text { versus } \\
4(0.6)\end{array}$ \\
\hline
\end{tabular}

Note: *Statistically significant.

Abbreviations: FREEDOM, Fracture Reduction Evaluation of Denosumab in Osteoporosis Every 6 Months trial; STAND, Study of Transitioning from Alendronate to Denosumab; HALT, Hormone Ablation Bone Loss Trial; PO, oral; AEs, adverse events; n, number.

steroidal AI therapy; and (2) femoral-neck BMD in patients aged $\geq 65$ years at baseline. ${ }^{53}$

Denosumab at $60 \mathrm{mg}$ SC every 6 months is currently the only FDA-approved antiresorptive therapy for the treatment of AI-induced bone loss in women with early-stage breast cancer. However, several guidelines, including those from the American Society of Clinical Oncology and the NCCN, recommend either a bisphosphonate or denosumab as appropriate therapy for those with documented osteoporosis (T-score $\leq-2.5$ ) or significant osteopenia (T-score $\leq-2.0$ ) and an increased fracture risk. ${ }^{18,19}$ The European Society for Medical Oncology recognizes that bisphosphonates prevent bone loss in patients with iatrogenic premature menopause and in postmenopausal patients treated with AIs, but the organization does not make specific recommendations about treatment. $^{20}$

\section{Denosumab and CTIBL in prostate cancer}

In the denosumab Hormone Ablation Bone Loss Trial (HALT), 1468 men receiving ADT (bilateral orchiectomy or gonadotropin-releasing hormone agonist) for nonmetastatic prostate cancer were randomly assigned to denosumab (60 mg SC every 6 months) or placebo. ${ }^{54}$ The primary endpoint was change in BMD at the lumbar spine at 24 months. Eligibility included men $\geq 70$ years of age, or men $<70$ years of age with baseline osteopenia (T-score at the lumbar spine, total hip, or femoral neck of $<-1.0)$. Key exclusion criteria included concurrent chemotherapy or radiation, prostatespecific antigen (PSA) $>5 \mathrm{ng} / \mathrm{mL}$ after 1 month of ADT, current or long-term prior bisphosphonate use, and a BMD T-score $<-4.0$. At 24 months, denosumab was associated with increased BMD at all measured sites, including the lumbar spine, total hip, femoral neck, distal third of the radius, and whole body (absolute difference versus placebo, 6.7\%, $4.8 \%, 3.9 \%, 5.5 \%$, and $4.0 \%$, respectively; $P<0.001$ for all comparisons). A significant increase in BMD was observed in all patient subgroups. With a sample size of almost 1500 patients, this study was powered to capture a difference in fracture rate, and a statistically significant decrease in new vertebral fractures was observed at 36 months in the denosumab arm (1.5\% versus $3.9 \%$ with placebo, relative risk $=0.38 ; 95 \% \mathrm{CI}=0.19-0.78 ; P=0.006)$. Fractures at any site also occurred in fewer denosumab-treated patients compared with the placebo group (38 patients [5.2\%] versus 53 patients [7.2\%]); however, this did not reach statistical significance $(P=0.10)$. In addition, denosumab significantly decreased the number of patients developing more than one fracture (denosumab, five patients [0.7\%] versus placebo, 18 patients $[2.5 \%], P=0.006)$. AE rates were similar between the groups, with no cases of ONJ reported in either arm (Table 2).

The NCCN recommends that men undergoing ADT be treated according to the National Osteoporosis Foundation guidelines as detailed above, with treatment recommended in patients with osteoporosis or osteopenia at high risk for fracture as evaluated by their FRAX score. ${ }^{6,24}$ Recommended treatment options include denosumab (60 mg SC every 6 months), zoledronic acid (5 mg IV annually), or alendronate (70 mg PO weekly). ${ }^{24}$ 


\section{Denosumab dosing}

Denosumab absorption is rapid and sustained, with a bioavailability of $62 \%-64 \%$, a steady-state mean serum concentration of $20.5 \mu \mathrm{g} / \mathrm{mL}$, and an elimination half-life of 28 days. ${ }^{55,56} \mathrm{~A}$ decrease in bone-resorption markers is observed within 24 hours after denosumab administration, and steady-state levels are achieved after 6 months of multiple doses at $120 \mathrm{mg}$ per month. ${ }^{48,55}$ The initial Phase I trial in healthy postmenopausal women demonstrated that a single denosumab dose of $3.0 \mathrm{mg} / \mathrm{kg}$ could suppress markers of bone turnover, including urinary collagen type 1 cross-linked N-telopeptide (uNTx), up to $80 \%$ for several months. ${ }^{48}$ A subsequent Phase II trial in patients with bone metastases from solid tumors with elevated uNTx:Cr levels despite IV bisphosphonate therapy compared denosumab (30-180 mg administered every 4 or 12 weeks) with continued IV bisphosphonate therapy. ${ }^{57}$ In this study, $71 \%$ of patients who switched to denosumab treatment had a decline in uNTx:Cr ratio compared with $29 \%$ of patients who continued therapy with IV bisphosphonates $(P<0.001)$, providing initial evidence that denosumab may be superior to IV bisphosphonates at suppressing bone resorption. ${ }^{57} \mathrm{~A}$ second Phase II trial in patients with metastatic breast cancer treated with multiple doses and schedules of denosumab confirmed a greater reduction in uNTx:Cr levels in denosumab-treated compared to IV bisphosphonate-treated patients (74\% versus $63 \%) .{ }^{58}$ In a recently published meta-analysis of multiple Phase I, Phase II, and Phase III trials evaluating the pharmacokinetics of denosumab in more than 1500 healthy subjects and postmenopausal women with low BMD, a fixed dose of denosumab at $60 \mathrm{mg}$ (the recommended dose for CTIBL) provided similar RANKL inhibition to that achieved with weight-based dosing. ${ }^{56}$ Importantly, patient age and race had little impact on denosumab's pharmacokinetics. Denosumab has not been studied in the pediatric population.

Denosumabelimination occurs through the immunoglobulin clearance pathway via the reticuloendothelial system, similar to that of other monoclonal antibodies, and is thus thought to be independent of renal or hepatic function. ${ }^{59}$

Dose reductions and renal monitoring are not required with denosumab therapy; however, there is a lack of safety data in patients with severe renal dysfunction. In the only published trial of denosumab use in renal impairment, a single $60 \mathrm{mg}$ dose was given to 55 patients, including 17 patients having severe chronic renal disease $(\mathrm{Cr}$ clearance $\leq 30 \mathrm{~mL} /$ minute) or requiring hemodialysis. Pharmacokinetics and changes in biomarkers of bone resorption were unaffected by renal function. ${ }^{60}$ Hypocalcemia was observed more frequently in patients with renal dysfunction with hypocalcemia of any grade in eight $(15 \%)$ patients and severe hypocalcemia requiring hospitalization in two (4\%) patients. ${ }^{60}$ The two patients with severe hypocalcemia had advanced renal disease and were enrolled before the protocol required baseline assessment of parathyroid hormone, calcium, and vitamin D. Thus, more frequent monitoring of serum calcium levels and the ensuring of adequate vitamin $\mathrm{D}$ levels prior to initiating denosumab therapy is recommended in this population.

\section{Denosumab side effects}

Much of what is known about the side effects of denosumab comes from its use in the setting of primary osteoporosis and in patients with bone metastasis.

The FREEDOM trial is the largest single trial comparing denosumab to placebo for the prevention of fractures in primary osteoporosis. ${ }^{49}$ In this study, there were no significant differences between the 3900 subjects who received denosumab and those who received placebo in the total incidence of AEs, serious adverse events (SAEs), or discontinuation of study treatment because of AEs. ${ }^{49}$ In addition, there was no increase in the risk of cancer, cardiovascular disease, delayed fracture healing, or hypocalcemia, and there were no cases of osteonecrosis of the jaw. Cellulitis was more frequently observed in the denosumab group, although there was no difference in the overall rate of infections. Neutralizing antibodies against denosumab were not identified. As summarized in Table 2, a similar safety profile was observed in the trials using denosumab to treat CTIBL in the settings of breast and prostate cancer.

Three international Phase III randomized, doubleblind, double-dummy, active-controlled studies comparing denosumab (120 mg SC every 4 weeks) with zoledronic acid (4 mg IV every 4 weeks) for the prevention of SREs in more than 5700 patients with bone metastases have been completed. ${ }^{61-63}$ These three registration trials were of identical design and focused on the prevention of SREs in patients with breast cancer, prostate cancer, and other solid tumors or multiple myeloma and lead to the FDA approval of denosumab for this indication. In a meta-analysis of these trials, the incidence of all AEs and SAEs was similar between treatment groups. ${ }^{64}$ However, as in the individual trials, there was an increased incidence of hypocalcemia (9.6\% versus $5.0 \%)$ and acute phase reactions (20.2\% versus $8.7 \%$ ) in the denosumab group compared with the zoledronic acid group, respectively. 
Incidence of ONJ was infrequent and similar between treatment groups, with a cumulative incidence of $1.3 \%$ (37 events) in the zoledronic acid group compared with $1.8 \%$ (52 events) in the denosumab group $(P=0.13)$.

Recently, there have been reports of atypical femur fractures in patients treated with denosumab. ${ }^{55,65}$ Atypical subtrochanteric and diaphyseal femur fractures, initially reported in patients using bisphosphonates long term ( $>5$ years), have a characteristic prodrome of mid-thigh pain, which may be accompanied by cortical thickening on plain radiographs obtained prior to fracture. The rate of atypical femur fractures in patients treated with both bisphosphonates and denosumab is much lower than the fracture risk associated with untreated osteoporosis.

As RANKL is expressed on subsets of T and B cells, there is a theoretical possibility that denosumab may be immunosuppressive. RANKL-deficient mice lack normal lymph node development and have inhibition of early $\mathrm{T}$ - and B-lymphocyte development. ${ }^{66}$ However, in clinical trials, a statistically significant or clinically meaningful effect on the immune system has not been observed to date. In one early trial of denosumab therapy, there was no significant effect on mean white blood cell counts, absolute lymphocyte counts, T-cell or B-cell counts, or immunoglobulins, nor was there a meaningful difference in incidence of infection. ${ }^{48}$ As noted above, trials of denosumab for the treatment and prevention of osteoporosis suggested a slight increase in the rate of certain infectious complications, including cellulitis. ${ }^{49,67}$ However, the overall infection rate did not differ significantly from placebo, and an association between denosumab and infectious SAEs was not observed in the three large Phase III registrational trials comprising over 5700 patients with metastatic cancer. ${ }^{64}$

\section{Sequential use}

The safety of switching from bisphosphonates to denosumab therapy has been studied in the treatment of patients with primary osteoporosis as well as in the setting of metastatic disease to prevent SREs, but not specifically in the setting of CTIBL. ${ }^{68-70}$ The Phase III Study of Transitioning from Alendronate to Denosumab trial evaluated the sequential use of alendronate followed by denosumab in postmenopausal women with primary osteoporosis. ${ }^{68}$ Eligible patients were postmenopausal women at least 55 years of age who had a lumbar spine or total hip BMD T-score of -2.0 to -4.0 and who had been receiving alendronate at $70 \mathrm{mg} /$ week for at least 6 months. Subjects were randomized to denosumab (60 mg SC every 6 months) versus continued oral alendronate.
The primary endpoint was change in total hip BMD after 12 months of therapy. BMD at the total hip increased significantly in patients transitioned to denosumab (1.90\%; $95 \% \mathrm{CI}=1.61 \%-2.18 \%$ ) compared with patients continuing on alendronate $(1.05 \% ; 95 \% \mathrm{CI}=0.76 \%-1.34 \%)(P<0.0001)$. These trials suggest a role for switching to denosumab in patients who are currently receiving bisphosphonate therapy and who continue to have a decline in BMD on DXA or have a persistently elevated uNTx level. Other indications for switching to denosumab might include progressive renal insufficiency, intolerance to bisphosphonate side effects, or the necessity for concomitant use of nephrotoxic medications. No data support the combined use of a bisphosphonate plus denosumab to further reduce osteoclast activity. As both agents are extremely potent osteoclast inhibitors, the risk of increased toxicity with combination therapy, especially with regard to ONJ development and hypocalcemia, would be particularly concerning.

\section{Future uses}

The use of bone-modifying agents in the prevention of cancer, cancer recurrence, and/or cancer progression is currently an area of active research. Several trials in early-stage breast cancer patients suggest a role for bone-modifying agents in improving disease-free survival. The Austrian Breast and Colorectal Cancer Study Group Trial-12 in premenopausal women with early-stage breast cancer is the most quoted of the recent trials in support of a role for bone-targeted therapy in the prevention of breast cancer recurrence. ${ }^{71}$ In this trial, patients treated with three years of $4 \mathrm{mg}$ IV zoledronic acid twice yearly had a $36 \%$ lower incidence of breast cancer recurrence compared with patients treated with hormonal therapy alone. Unfortunately, the much larger Adjuvant Zoledronic Acid to Reduce Recurrence trial performed in higher-risk early-stage breast cancer patients with stage 2 and 3 disease treated predominantly with chemotherapy alone versus chemotherapy plus higher-intensity zoledronic acid failed to show a similar improvement in breast cancer recurrence. ${ }^{72}$ However, in a subgroup analysis there was an improvement in both disease-free and overall survival in older women $(>5$ years postmenopausal or $>60$ years of age) treated with zoledronic acid. Several other trials have also recently been presented that offer additional conflicting results about the effectiveness of intravenous bisphosphonates in preventing breast cancer recurrence in patients with earlystage disease (reviewed in Wong et al). ${ }^{73}$ One hypothesis for the results of the Austrian Breast and Colorectal Cancer Study Group Trial-12 and Adjuvant Zoledronic Acid to Reduce 
Recurrence trials is that bisphosphonates play a role in the prevention of breast cancer recurrence, but only in very-lowestrogen states. ${ }^{71,72}$ However, the exact mechanism or effect bisphosphonates play in this setting is still controversial.

The RANK-RANKL system is also implicated in mammary epithelial proliferation and carcinogenesis. In murine models, overexpression of RANK promotes hormone- and carcinogen-induced murine mammary tumor formation, whereas pharmacologic inhibition of RANKL attenuates tumor development. ${ }^{74}$ This and other animal data suggest that there may be a role for RANKL inhibitors like denosumab in both chemoprevention and the prevention of cancer recurrence through direct effects on breast tissue and breast cancer stem cells. ${ }^{74-76}$ Similarly, there is data supporting an integral role for RANK-RANKL in the spread and propagation of cancer cells in bone, helping to explain the osteotropism of tumors, including prostate, breast, and lung cancer, that express RANK. ${ }^{77}$ Denosumab may have the potential to interrupt the "vicious cycle" of cancer-induced bone destruction and tumor cell expansion and to inhibit breast cancer stem cell survival. ${ }^{78}$ The international Phase III Denosumab as Adjuvant Treatment for Women With High Risk Early Breast Cancer Receiving Neoadjuvant or Adjuvant Therapy (D-CARE) trial has recently completed accrual and is powered to assess the effect of denosumab on disease recurrence in patients with stage 2 and 3 high-risk early stage breast cancer. ${ }^{79}$ The Austrian Breast and Colorectal Cancer Study Group Study 18 trial is currently enrolling patients and will similarly evaluate the effect of denosumab on the time to first clinical fracture and bone metastasis, disease-free survival, and overall survival. ${ }^{52}$

The role of denosumab in delaying the development of bone metastasis has been demonstrated in the prostate cancer 147 trial ${ }^{80}$ In this Phase III double blind, placebo-controlled trial, 716 men with castrate-resistant prostate cancer at high risk for the development of bone metastases (PSA $>8.0 \mathrm{ug} / \mathrm{L}$ or PSA doubling time $<10.0$ months) were randomized to denosumab $120 \mathrm{mg}$ SC or SC placebo every 4 weeks. ${ }^{81}$ The primary end point of bone metastasis-free survival time was 25.2 months in the placebo group compared with 29.5 months in the denosumab group (hazard ratio $=0.85$; $95 \% \mathrm{CI}=0.73-0.98 ; P=0.028)$, with those at greatest risk of metastases (PSA doubling time $\leq 6$ months) having the greatest benefit. As overall survival was similar between the two groups, it is currently unclear if denosumab merely delayed rather than prevented bone metastases in this patient population. The FDA declined to approve denosumab for this indication.
The role of denosumab in preventing or delaying cancer development and progression awaits the results of the D-CARE and Austrian Breast and Colorectal Cancer Study Group Study 18 trials as well as further follow-up of the 147 prostate cancer trial.

\section{Conclusion}

Attention to bone health is an essential component of cancer care, particularly in women and men undergoing hormone ablation therapy for the treatment of breast and prostate cancer. Oral bisphosphonates remain the most commonly used therapeutic option in this setting, but additional treatment options are required for patients who do not respond adequately or are intolerant to bisphosphonates, have renal insufficiency, or require treatment with nephrotoxic medications. For these patients, denosumab offers an effective and well-tolerated alternative. In addition, bisphosphonates are retained in the bone for decades and the long-term implications, especially in younger patients, are unknown. As with bisphosphonates, data regarding the long-term toxicities of denosumab are lacking. However denosumab's shorter half-life and reversibility are attractive when considering long-term use or therapy for younger patients. The choice between bisphosphonates and denosumab will become better informed as the role these agents play in cancer progression, recurrence, and prevention is established. The added cost of denosumab as a novel and newly approved biologic agent, particularly in comparison with the notably lower costs of generic bisphosphonates, must also be considered when recommending a therapy. The data presented highlight the essential role that bonemodifying agents play in maintaining bone health in patients undergoing cancer care and the emerging role of denosumab for this indication.

\section{Disclosure}

A Stopeck has consulted, received honoraria, and received research and clinical trial support from Amgen, received honoraria from GlaxoSmithKline, and received clinical trial support from Novartis. U Brown-Glaberman reports no conflict of interest in this work. The authors report no other conflicts of interest in this work.

\section{References}

1. NIH Consensus Development Panel on Osteoporosis Prevention, Diagnosis, and Therapy. Osteoporosis prevention, diagnosis and therapy. JAMA. 2001;285(6):785-795.

2. Lui H, Paige NM, Goldzweig CL, et al. Screening for osteoporosis in men: a systematic review for an American College of Physicians guideline. Ann Intern Med. 2008;148(9):685-701. 
3. Prevention and management of osteoporosis. World Health Organ Tech Rep Ser. 2003;921:1-164.

4. World Health Organization. FRAX ${ }^{\circledR}$ WHO fracture risk assessment tool: welcome to $\mathrm{FRAX}^{\circledR}$ [homepage on the Internet]. Sheffield, United Kingdom: World Health Organization Collaborating Centre for Metabolic Bone Diseases; 2013. Available from: http://www.sheffield. ac.uk/FRAX/. Accessed February 5, 2013.

5. Hadji P, Aapro MS, Body JJ, et al. Management of aromatase inhibitorassociated bone loss in postmenopausal women with breast cancer: practical guidance for prevention and treatment. Ann Oncol. 2011; 22(12):2546-2555.

6. National Osteoporosis Foundation. 2013 clinician's guide to the prevention and treatment of osteoporosis [webpage on the Internet]. Washington, DC: National Osteoporosis Foundation; 2013. Available from: http://nof.org/hcp/resources/913. Accessed February 10, 2013.

7. Bolland MJ, Avenell A, Baron JA, et al. Effect of calcium supplements on risk of myocardial infarction and cardiovascular events: meta-analysis. BMJ. 2010;341:c3691.

8. Bolland MJ, Grey A, Avenell A, Gamble GD, Reid IR. Calcium supplements with or without vitamin D and risk of cardiovascular events: reanalysis of the Women's Health Initiative limited access dataset and meta-analysis. BMJ. 2011;342:d2040.

9. Kearns AE, Khosla S, Kostenuik PJ. Receptor activator of nuclear factor kappaB ligand and osteoprotegerin regulation of bone remodeling in health and disease. Endocr Rev. 2008;29(2):155-192.

10. Manolagas SC, Kousteni S, Jilka RL. Sex steroids and bone. Recent Prog Horm Res. 2002;57:385-409.

11. Khosla S, Melton LJ 3rd, Riggs BL. The unitary model for estrogen deficiency and the pathogenesis of osteoporosis: is a revision needed? J Bone Miner Res. 2011;26(3):441-451.

12. Khosla S. Update on estrogens and the skeleton. J Clin Endocrinol Metab. 2010;95(8):3569-3577.

13. Khosla S, Amin S, Orwoll E. Osteoporosis in men. Endocr Rev. 2008;29(4):441-464.

14. Lorentzon M, Swanson C, Andersson N, Mellström D, Ohlsson C. Free testosterone is a positive, whereas free estradiol is a negative, predictor of cortical bone size in young Swedish men: the GOOD study. $J$ Bone Miner Res. 2005;20(8):1334-1341.

15. Eastell R, Adams JE, Coleman RE, et al. Effect of anastrozole on bone mineral density: 5-year results from the anastrozole, tamoxifen, alone or in combination trial 18233230. J Clin Oncol. 2008;26(7):1051-1057.

16. Forbes JF, Cuzick J, Buzdar A, Howell A, Tobias JS, Baum M; for Arimidex, Tamoxifen, Alone or in Combination (ATAC) Trialists' Group. Effect of anastrozole and tamoxifen as adjuvant treatment for early-stage breast cancer: 100-month analysis of the ATAC trial. Lancet Oncol. 2008;9(1):45-53.

17. Amir E, Seruga B, Niraula S, Carlsson L, Ocaña A. Toxicity of adjuvant endocrine therapy in postmenopausal breast cancer patients: a systematic review and meta-analysis. J Natl Cancer Inst. 2011; 103(17):1299-1309.

18. Hillner BE, Ingle JN, Chlebowski RT, et al; for American Society of Clinical Oncology. American Society of Clinical Oncology 2003 update on the role of bisphosphonates and bone health issues in women with breast cancer. J Clin Oncol. 2003;21(21):4042-4057.

19. Gralow JR, Biermann JS, Farooki A, et al. NCCN Task Force report: bone health in cancer care. J Natl Compr Canc Netw. 2009;7 Suppl 3: S1-S32; quiz S33-S35.

20. Aebi S, Davidson T, Gruber G, Cardoso F; for EMSO Guidelines Working Group. Primary breast cancer: ESMO Clinical Practice Guidelines for diagnosis, treatment and follow-up. Ann Oncol. 2011;22 Suppl 6: vi12-vi24.

21. Saad F, Adachi JD, Brown JP, et al. Cancer treatment-induced bone loss in breast and prostate cancer. J Clin Oncol. 2008;26(33): 5465-5476.

22. Krupski TL, Smith MR, Lee WC, et al. Natural history of bone complications in men with prostate carcinoma initiating androgen deprivation therapy. Cancer. 2004;101(3):541-549.
23. Shahinian VB, Kuo YF, Freeman JL, Goodwin JS. Risk of fracture after androgen deprivation for prostate cancer. $N$ Engl J Med. 2005;352(2): 154-164.

24. National Comprehensive Cancer Network. NCCN Clinical Practice Guidelines in Oncology (NCCN Guideline $\left.{ }^{\circledR}\right)$ : Prostate Cancer. V.2.2013. Fort Washington, PA: National Comprehensive Cancer Network; 2013. Available from: http://www.nccn.org/professionals/physician_gls/pdf/ prostate.pdf. Accessed February 8, 2013.

25. Cabanillas ME, Lu H, Fang S, Du XL. Elderly patients with non-Hodgkin lymphoma who receive chemotherapy are at higher risk for osteoporosis and fractures. Leuk Lymphoma. 2007;48(8): $1514-1521$.

26. Hu MI, Lu H, Gagel RF. Cancer therapies and bone health. Curr Rheumatol Rep. 2010;12(3):177-185.

27. Shapiro CL, Manola J, Leboff M. Ovarian failure after adjuvant chemotherapy is associated with rapid bone loss in women with earlystage breast cancer. J Clin Oncol. 2001;19(14):3306-3311.

28. Nilsson OS, Bauer HC, Brostrom LA. Comparison of the effects of adriamycin and methotrexate on orthotopic and induced heterotopic bone in rats. J Orthop Res. 1990;8(2):199-204.

29. Wang TM, Shih C. Study of histomorphometric changes of the mandibular condyles in neonatal and juvenile rats after administration of cyclophosphamide. Acta Anat (Basel). 1986;127(2):93-99.

30. Köther M, Schindler J, Oette K, Berthold F. Abnormalities in serum osteocalcin values in children receiving chemotherapy including ifosfamide. In Vivo. 1992;6(2):219-221.

31. Von Hoff DD, Schilsky R, Reichert CM, et al. Toxic effects of cisdichlorodiammineplatinum(II) in man. Cancer Treat Rep. 1979; 63(9-10):1527-1531.

32. Warner JT, Evans WD, Webb DK, Bell W, Gregory JW. Relative osteopenia after treatment for acute lymphoblastic leukemia. Pediatr Res. 1999;45(4 Pt 1):544-551.

33. Jia D, O’Brien CA, Stewart SA, Manolagas SC, Weinstein RS. Glucocorticoids act directly on osteoclasts to increase their life span and reduce bone density. Endocrinology. 2006;147(12):5592-5599.

34. Hofbauer LC, Gori F, Riggs BL, et al. Stimulation of osteoprotegerin ligand and inhibition of osteoprotegerin production by glucocorticoids in human osteoblastic lineage cells: potential paracrine mechanisms of glucocorticoid-induced osteoporosis. Endocrinology. 1999;140(10): 4382-4389.

35. Gandhi MK, Lekamwasam S, Inman I, et al. Significant and persistent loss of bone mineral density in the femoral neck after haematopoietic stem cell transplantation: long-term follow-up of a prospective study. Br J Haematol. 2003;121(3):462-468.

36. Tauchmanovà L, Serio B, Del Puente A, et al. Long-lasting bone damage detected by dual-energy $\mathrm{x}$-ray absorptiometry, phalangeal osteosonogrammetry, and in vitro growth of marrow stromal cells after allogeneic stem cell transplantation. J Clin Endocrinol Metab. 2002; 87(11):5058-5065.

37. Truven Health Analytics. Micromedex gateway [webpage on the Internet]. Greenwood Village, CO: Truven Health Analytics Inc; 2012-2013. Available from: http://www.micromedexsolutions.com/ micromedex2/librarian/. Accessed May 15, 2013.

38. McClung M, Harris ST, Miller PD, et al. Bisphosphonate therapy for osteoporosis: benefits, risks, and drug holiday. Am J Med. 2013;126(1): $13-20$.

39. Brufsky A, Harker WG, Beck JT, et al. Zoledronic acid inhibits adjuvant letrozole-induced bone loss in postmenopausal women with early breast cancer. J Clin Oncol. 2007;25(7):829-836.

40. Bundred NJ, Campbell ID, Davidson N, et al. Effective inhibition of aromatase inhibitor-associated bone loss by zoledronic acid in postmenopausal women with early breast cancer receiving adjuvant letrozole: ZO-FAST Study results. Cancer. 2008;112(5):1001-1010.

41. Llombart A, Frassoldati A, Paija O, et al. Immediate administration of zoledronic acid reduces aromatase inhibitor-associated bone loss in postmenopausal women with early breast cancer: 12 -month analysis of the E-ZO-FAST trial. Clin Breast Cancer. 2012;12(1):40-48. 
42. Brufsky AM, Harker WG, Beck JT, et al. Final 5-year results of Z-FAST trial: adjuvant zoledronic acid maintains bone mass in postmenopausal breast cancer patients receiving letrozole. Cancer. 2012;118(5): 1192-1201.

43. Van Poznak C, Hannon RA, Mackey JR, et al. Prevention of aromatase inhibitor-induced bone loss using risedronate: the SABRE trial. J Clin Oncol. 2010;28(6):967-975.

44. Smith MR, McGovern FJ, Zietman AL, et al. Pamidronate to prevent bone loss during androgen-deprivation therapy for prostate cancer. N Engl J Med. 2001;345(13):948-955.

45. Smith MR, Eastham J, Gleason DM, Shasha D, Tchekmedvian S, Zinner N. Randomized controlled trial of zoledronic acid to prevent bone loss in men receiving androgen deprivation therapy for nonmetastatic prostate cancer. J Urol. 2003;169(6):2008-2012.

46. Greenspan SL, Nelson JB, Trump DL, Resnick NM. Effect of onceweekly oral alendronate on bone loss in men receiving androgen deprivation therapy for prostate cancer: a randomized trial. Ann Intern Med. 2007;146(6):416-424.

47. Klotz LH, McNeill IY, Kebabdjian M, Zhang L, Chin J; for Canadian Urology Research Consortium. A phase 3, double-blind, randomised, parallel-group, placebo-controlled study of oral weekly alendronate for the prevention of androgen deprivation bone loss in nonmetastatic prostate cancer: the cancer and osteoporosis research with alendronate and leuprolide (CORAL) study. Eur Urol. 2013;63(5):927-935.

48. Bekker PJ, Holloway DL, Rasmussen AS, et al. A single-dose placebocontrolled study of AMG 162, a fully human monoclonal antibody to RANKL, in postmenopausal women. 2004. J Bone Miner Res. 2005;20(12):2275-2282.

49. Cummings SR, San Martin J, McClung MR, et al; for FREEDOM Trial. Denosumab for prevention of fractures in postmenopausal women with osteoporosis. N Engl J Med. 2009;361(8):756-765.

50. Brown JP, Prince RL, Deal C, et al. Comparison of the effect of denosumab and alendronate on BMD and biochemical markers of bone turnover in postmenopausal women with low bone mass: a randomized, blinded, phase 3 trial. J Bone Miner Res. 2009;24(1): $153-161$.

51. Ellis GK, Bone HG, Chlebowski R, et al. Randomized trial of denosumab in patients receiving adjuvant aromatase inhibitors for nonmetastatic breast cancer. J Clin Oncol. 2008;26(30):4875-4882.

52. Amgen. Study to determine treatment effects of denosumab in patients with breast cancer receiving aromatase inhibitor therapy. In: ClinicalTrials.gov [website on the Internet]. Bethesda, MD: US National Library of Medicine; 2013 [updated March 15, 2013]. Available from: http://clinicaltrials.gov/ct2/show/NCT00556374. Accessed February 15, 2013.

53. Ellis GK, Bone HG, Chlebowski R, et al. Effect of denosumab on bone mineral density in women receiving adjuvant aromatase inhibitors for non-metastatic breast cancer: subgroup analyses of a phase 3 study. Breast Cancer Res Treat. 2009;118(1):81-87.

54. Smith MR, Egerdie B, Hernández Toriz N, et al; for Denosumab HALT Prostate Cancer Study Group. Denosumab in men receiving androgen-deprivation therapy for prostate cancer. $N$ Engl $J$ Med. 2009;361(8):745-755.

55. XGEVA ${ }^{\circledR}$ (denosumab) [package insert]. Thousand Oaks, CA: Amgen Inc; 2010

56. Sutjandra L, Rodriguez RD, Doshi S, et al. Population pharmacokinetic meta-analysis of denosumab in healthy subjects and postmenopausal women with osteopenia or osteoporosis. Clin Pharmacokinet. 2011;50(12):793-807.

57. Fizazi K, Lipton A, Mariette X, et al. Randomized phase II trial of denosumab in patients with bone metastases from prostate cancer, breast cancer, or other neoplasms after intravenous bisphosphonates. J Clin Oncol. 2009;27(10):1564-1571.

58. Lipton A, Steger CG, Figueroa J, et al. Randomized active-controlled phase II study of denosumab efficacy and safety in patients with breast cancer-related bone metastases. J Clin Oncol. 2007;25(28): $4431-4437$.
59. Tabrizi MA, Tseng CM, Roskos LK. Elimination mechanisms of therapeutic monoclonal antibodies. Drug Discov Today. 2006; $11(1-2): 81-88$.

60. Block GA, Bone HG, Fang L, Lee E, Padhi D. A single-dose study of denosumab in patients with various degrees of renal impairment. $J$ Bone Miner Res. 2012;27(7):1471-1479.

61. Stopeck AT, Lipton A, Body JJ, et al. Denosumab compared with zoledronic acid for the treatment of bone metastases in patients with advanced breast cancer: a randomized, double-blind study. J Clin Oncol. 2010;28(35):5132-5139.

62. Fizazi K, Carducci M, Smith M, et al. Denosumab versus zoledronic acid for treatment of bone metastases in men with castration-resistant prostate cancer: a randomised, double-blind study. Lancet. 2011; 377(9768):813-822.

63. Henry DH, Costa L, Goldwasser F, et al. Randomized, double-blind study of denosumab versus zoledronic acid in the treatment of bone metastases in patients with advanced cancer (excluding breast and prostate cancer) or multiple myeloma. J Clin Oncol. 2011;29(9):1125-1132.

64. Lipton A, Fizazi K, Stopeck AT, et al. Superiority of denosumab to zoledronic acid for prevention of skeletal-related events: a combined analysis of 3 pivotal, randomised, phase 3 trials. Eur J Cancer. 2012; 48(16):3082-3092.

65. Paparodis R, Buehring B, Pelley E, Binkley N. A case of unusual subtrochanteric fracture in a patient receiving denosumab. Endocr Pract. 2013:1-17. [Epub ahead of print].

66. Kong YY, Yoshida H, Sarosi I, et al. OPGL is a key regulator of osteoclastogenesis, lymphocyte development and lymph-node organogenesis. Nature. 1999;397(6717):315-323.

67. Miller PD, Wagman RB, Peacock M, et al. Effect of denosumab on bone mineral density and biochemical markers of bone turnover: six year results of a phase 2 clinical trial. J Clin Endocrinol Metab. 2011;96(2):394-402.

68. Kendler DL, Roux C, Benhamou CL, et al. Effects of denosumab on bone mineral density and bone turnover in postmenopausal women transitioning from alendronate therapy. J Bone Miner Res. 2010;25(1):72-81.

69. Fizazi K, Brown JE, Carducci M, et al. Denosumab in patients with metastatic prostate cancer previously treated with denosumab or zoledronic acid: 2-year open-label extension phase results from the pivotal phase 3 study. European Society for Medical Oncology 2012 Congress; September 28-October 2, 2012; Vienna, Austria. Abstract 937P.

70. Stopeck AT, Lipton A, Martín M, et al. Denosumab in patients with breast cancer and bone metastases previously treated with zoledronic acid or denosumab: results from the 2-year open-label extension treatment phase of a pivotal phase 3 study. 2011 CTRC-AACR San Antonio Breast Cancer Symposium;. December 6-10, 2011; San Antonio, TX. Abstract P3-16-07.

71. Gnant M, Mineritsch B, Schippinger W, et al; for ABCSG-12 Trial Investigators. Endocrine therapy plus zoledronic acid in premenopausal breast cancer. N Engl J Med. 2009;360(7):679-691.

72. Coleman RE, Marshall H, Cameron D, et al; for AZURE Investigators. Breast-cancer adjuvant therapy with zoledronic acid. $N$ Engl $J$ Med. 2011;365(15):1396-1405.

73. Wong MH, Stockler MR, Pavlakis N. Bisphosphonates and other bone agents for breast cancer. Cochrane Database Syst Rev. 2012;2: CD003474.

74. Gonzalez-Suarez E, Jacob AP, Jones J, et al. RANK ligand mediates progestin-induced mammary epithelial proliferation and carcinogenesis. Nature. 2010;468(7320):103-107.

75. Schramek D, Leibbrandt A, Sigl V, et al. Osteoclast differentiation factor RANKL controls development of progestin-driven mammary cancer. Nature. 2010;468(7320):98-102.

76. Gonzalez-Suarez E, Branstetter D, Armstrong A, Dinh H, Blumberg H, Dougall WC. RANK overexpression in transgenic mice with mouse mammary tumor virus promoter-controlled RANK increases proliferation and impairs alveolar differentiation in the mammary epithelia and disrupts lumen formation in cultured epithelial acini. Mol Cell Biol. 2007;27(4):1442-1454. 
77. Jones DH, Nakashima T, Sanchez OH, et al. Regulation of cancer cell migration and bone metastasis by RANKL. Nature. 2006;440(7084): 692-696.

78. Roodman GD. Mechanisms of bone metastasis. $N$ Engl J Med. 2004;350(16):1655-1664.

79. Amgen. Study of denosumab as adjuvant treatment for women with high risk early breast cancer receiving neoadjuvant or adjuvant therapy (D-CARE). In: ClinicalTrials.gov [website on the Internet] Bethesda, MD: US National Library of Medicine; 2013 [updated January 25, 2013]. Available from: http://clinicaltrials.gov/ct2/show/ NCT01077154. Accessed February 15, 2013.
80. Smith MR, Saad F, Coleman R, et al. Denosumab and bone-metastasisfree survival in men with castration-resistant prostate cancer: results of a phase 3, randomised, placebo-controlled trial. Lancet. 2012;379(9810): 39-46.

\section{Publish your work in this journal}

Clinical Pharmacology: Advances and Applications is an international, peer-reviewed, open access journal publishing original research, reports, reviews and commentaries on all areas of drug experience in humans. The manuscript management system is completely online and includes a very quick and fair peer-review system, which is all easy to use.
Visit http://www.dovepress.com/testimonials.php to read real quotes from published authors. 